\title{
Synthetic-Heterodyne Interferometric Demodulation
}

\author{
JAMES H. COLE, BRUCE A. DANVER, AND JOSEPH A. BUCARO
}

\begin{abstract}
A new interferometric demodulation technique is described. This technique employs phase swept multicarrier demodulation (synthetic-heterodyne) to optimize the interferometer output.
\end{abstract}

\section{INTRODUCTION}

$\mathrm{T}$ HE advent of fiber interferometric sensors [1] has produced increased interest in the optimization of interferometers to maintain the maximum output signal. Several optimization techniques are currently available: heterodyne-FM [2], phase locking [3]-[5], phase swept modulation [6] , [7], and frequency stabilization [8], [9]. Each of these techniques has disadvantages. Both the optical and electrical power required for the heterodyne technique exceed the power requirements of phase modulators. Phase lock techniques require a large dynamic range resulting in relatively large phase modulators and circuit resetting. The large modulators are undesirable in multisensor systems where phase locking requires one modulator per sensor. Phase swept modulation utilizes AM demodulation techniques and therefore is sensitive to amplitude (optical intensity) fluctuations. The frequency stabilization technique requires one laser per sensor resulting in high electrical power consumption for multisensor systems. Interferometric demodulation utilizing the electronic synthesis of a heterodyne signal from an induced phase modulation appears to resolve the above disadvantages and is particularly attractive for multisensor systems.

\section{THEORY}

A fiber interferometric sensor depends on detecting a phase shift induced by an appropriate field. The demodulation system described here employs a phase modulator to provide a carrier signal. Assuming an optical mixing efficiency of 1 , the output from an appropriately configured interferometer can be represented as

$$
S=A^{2}+B^{2}+2 A B \cos \left(\phi_{H} \sin \omega_{H} t+\phi_{A} \sin \omega_{A} t+\phi\right)
$$

where $A$ and $B$ are the amplitudes of the reference and sensor optical beams, respectively. $\phi_{H}(\sim 2.6 \mathrm{rad})$ is the amplitude of a pure sinusoid used to drive a phase modulator and $\omega_{H}$ is the carrier angular frequency. $\phi_{A}$ is the peak phase excursion of the desired signal, $\omega_{A}$ is the signal angular frequency, and $\phi$ includes all time varying and static phase drift terms. The third term of (1) can be expressed in terms of Bessel functions

Manuscript received August 28, 1981; revised October 6, 1981.

J. H. Cole and J. A. Bucaro are with the Acoustics Division, Naval Research Laboratory, Washington, DC, 20735.

B. A. Danver is with Sachs Freeman Associates, Bowie, MD 20715.

$$
\begin{aligned}
S(t)= & 2 A B\left\{\sum_{n=0}^{\infty} J_{2 n}\left(\phi_{H}\right) \cos 2 n \omega_{H} t \cos (\beta+\phi)\right. \\
& \left.-\sum_{n=0}^{\infty} J_{2 n+1}\left(\phi_{H}\right) \sin (2 n+1) \omega_{H} t \sin (\beta+\phi)\right\} \\
= & 2 A B\left\{J_{0}\left(\phi_{H}\right)-J_{1}\left(\phi_{H}\right) \sin \omega_{H} t \sin (\beta+\phi)\right. \\
& \left.+J_{2}\left(\phi_{H}\right) \cos 2 \omega_{H} t \cos (\beta+\phi)+\cdots\right\}
\end{aligned}
$$

where $\beta=\phi_{A}$ sin $\omega_{A} t$. This signal (2) is applied to the input of the synthetic-heterodyne circuit diagrammed in Fig. 1, and is filtered into carrier components at $\omega_{H}$ and $2 \omega_{H}$

$$
\begin{aligned}
& S_{1}(t)=-2 A B\left[J_{1}\left(\phi_{H}\right) \sin \omega_{H} t \sin (\beta+\phi)\right] \\
& S_{2}(t)=2 A B\left[J_{2}\left(\phi_{H}\right) \cos 2 \omega_{H} t \cos (\beta+\phi)\right] .
\end{aligned}
$$

Analysis of (3a) clearly demonstrates the nature of interferometer fading. As $\beta+\phi$ drifts from $0-2 \pi$ due to thermal or mechanical variations, the relative amplitude of the frequency components at $\omega_{H}$ and $2 \omega_{H}$ fade. If $\omega_{H}$ were the desired signal, and $\beta=0$, drifting of $\phi$ could actually drive $S_{1}(t)$ to zero. In this demodulation technique however, the desired signal $\beta$ is always present as a sideband to either $\omega_{H}$ or $2 \omega_{H}$, since both carriers components cannot fade to zero simultaneously. Synthetic-heterodyning processes each carrier and combines them to form a nonfading carrier. This is accomplished by multiplying the $\omega_{H}$ channel with a local oscillator at $2 \omega_{H}$ while the $2 \omega_{H}$ channel is multiplied with a local oscillator at $\omega_{H}$ resulting in the signals of (4). The two local oscillators are synthesized in phase by dividing down a $1 \mathrm{MHz}$ clock frequency and filtering as shown in Fig. 1.

$$
\begin{aligned}
& S_{1 A}(t)=2 A B J_{1}\left(\phi_{H}\right) \sin \omega_{H} t \sin (\beta+\phi) \cos \left(2 \omega_{H} t+\theta\right) \\
& S_{2 A}(t)=2 A B J_{2}\left(\phi_{H}\right) \cos 2 \omega_{H} t \cos (\beta+\phi) \cos \left(\omega_{H} t+\theta\right) .
\end{aligned}
$$

Here $\theta$ is static phase term representing the phase difference between local oscillators and the incoming phase modulated signals. The signals (4) are bandpassed at $3 \omega_{H}$ providing the outputs that may be represented by

$$
\begin{aligned}
& S_{1 A}(t)=-2 A B J_{1}\left(\phi_{H}\right) \sin \left(3 \omega_{H} t+\theta\right) \sin (\beta+\phi) \\
& S_{2 A}(t)=2 A B J_{2}\left(\phi_{H}\right) \cos \left(3 \omega_{H} t+\theta\right) \cos (\beta+\phi) .
\end{aligned}
$$

If $J_{1}\left(\phi_{H}\right)$ is equal to $J_{2}\left(\phi_{H}\right)$, which can be achieved by adjusting the amplitude of the modulator or by employing a relative gain difference between channels, the signals (5) can be added to produce a signal represented as 


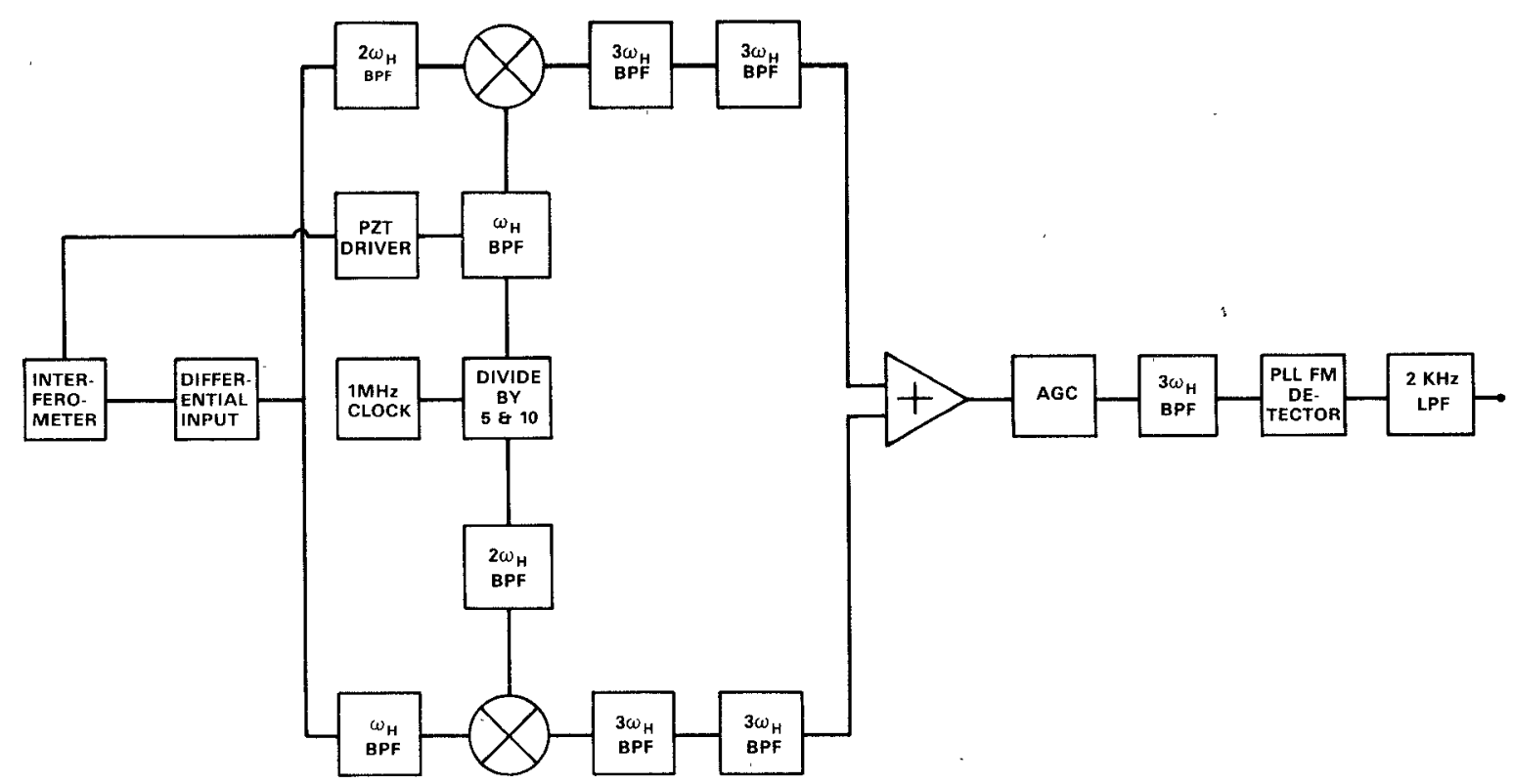

Fig. 1. Block diagram of the synthetic-heterodyne demodulator used in the experiment. $\mathrm{BPF}=$ bandpass filter, $\mathrm{LPF}=$ low-pass filter.

$$
\begin{aligned}
S_{A} & =S_{1 A}(t)+S_{2 A}(t) \\
& =2 A B J_{1}\left(\phi_{H}\right) \cos \left(3 \omega_{H} t+\theta+\beta+\phi\right) .
\end{aligned}
$$

$S_{A}$ is a heterodyne signal that can be demodulated using standard FM or PM techniques. A phase locked loop (PLL) FM discriminator is included in Fig. 1. As in standard FM limiting and demodulation techniques, signal fading is eliminated.

\section{EXPERIMENT}

The experimental configuration employed to demonstrate synthetic-heterodyne demodulation is illustrated in Fig. 2. A Tropel 100 laser beam is split by a cube beamsplitter, with each beam being focused into a single-mode fiber (ITT T-1601). One fiber forms the reference arm of the interferometer The other fiber contains a high frequency phase modulator [3], [4] which generates a carrier frequency, and a low frequency phase modulator to simulate the fiber sensor. The output of each fiber is collimated, combined on a plate beamsplitter, and directed to a photomultiplier tube (PMT). The electrical output of the PMT, which is proportional to the signal of (1), is applied to the input of the synthetic-heterodyne demodulator as illustrated in Fig. 1.

The operation of the synthetic-heterodyne demodulator is illustrated in the photographs of Figs. 3-5. Figs. 3 and 4 are photographs of the display of a Tektronix 7L5 spectrum analyzer with settings as indicated. The frequency components of the carriers are shown in Fig. 3. The amplitude at each frequency is arbitrary since all amplitudes were obtained with the analyzer in peak hold operation made over several scans. Fig. 3(a) and (b) shows the PMT output. In each case the frequencies $\omega_{H}, 2 \omega_{H}$, and higher harmonics are illustrated with no applied signal [Fig. 3(a)] and with a large amplitude $1 \mathrm{kHz}$ signal [Fig. 3(b)], respectively. The broadening of the $\omega_{H}$ and $2 \omega_{H}$ carriers [Fig. 3(b)] clearly demonstrates the sidebands around each carrier frequency utilized in synthetic-

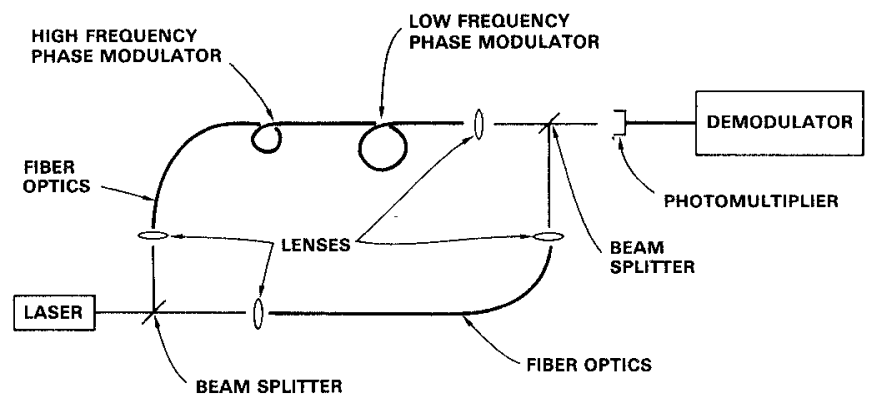

Fig. 2. Interferometer configuration employed for demonstrating synthetic-heterodyne.

heterodyning. The output from the discriminator indicates low harmonic distortion with the second harmonic approximately $38 \mathrm{~dB}$ down from the first (Fig. 4). The analyzer output of Fig. 4 is single scan, therefore, unlike Fig. 3, the amplitudes are calibrated. In fact, the peak at $1 \mathrm{kHz}$ represents a phase shift of $2.33 \mathrm{rad}$. The peak phase shift was determined by monitoring the PMT output with no carrier signal applied to the high frequency modulator but with the low frequency modulator operational. The peak amplitudes at each harmonic of the $1 \mathrm{kHz}$ signal were measured and from the ratios to the peak amplitude at $1 \mathrm{kHz}$, the phase shift was determined from a table of Bessel functions." The signal-to-noise ratio of Fig. 4 indicates that the minimum detectable phase shift is approximately $10^{-3}$ rad. Although this detection threshold is 3 orders of magnitude higher than has been demonstrated for phaselocked techniques [3], the authors believe that it is excellent for the preliminary demonstration of synthetic-heterodyning discussed here. This conclusion results from the fact that precise phase matching of the $\omega_{H}$ and the $2 \omega_{H}$ channels (Fig. 2 ) is required for optimum performance. Such phase matching must be compensated to minimize environmental effects on the demodulator performance. For this initial demonstration, minimal emphasis was given to these phase compensation 


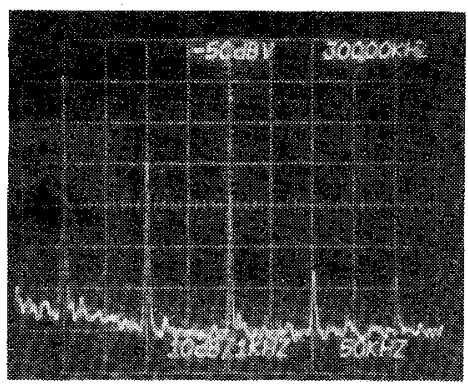

(a)

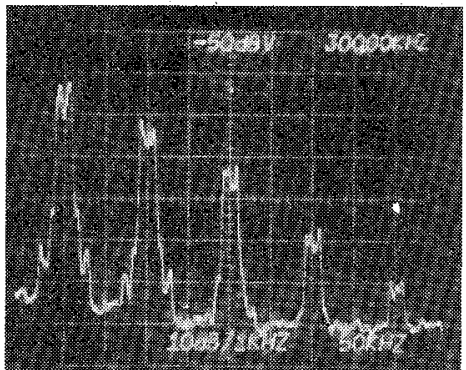

(b)

Fig. 3. Synthetic-heterodyne carrier and harmonics. (a) Without applied signal. (b) With a large amplitude $1 \mathrm{kHz}$ applied signal. (Uncalibrated amplitudes, $300 \mathrm{kHz}$ center screen).

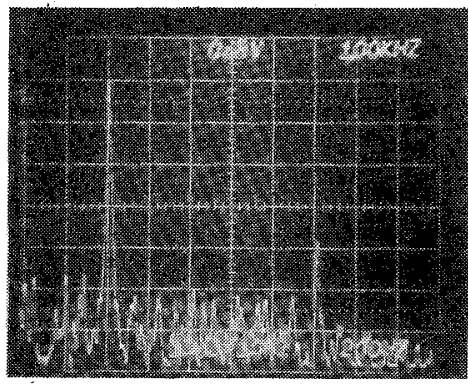

Fig. 4. Demodulated output and second harmonic (1 $\mathrm{kHz}$ is two large divisions from left).

requirements. As in conventional FM systems, syntheticheterodyning is relatively insensitive to amplitude fluctuations. For example, conventional FM radio is insensitive to signal fading when the signal is above a given threshold-level; similarly, synthetic-heterodyning is relatively immune to signal fading from amplitude (optical intensity) fluctuations. This insensitivity is illustrated in Fig. 5, where a reduction of 30 percent in carrier level does not affect the demodulated output. An improved design should provide a carrier dynamic range of at least $50 \mathrm{~dB}$.

\section{CONCLUSIONS}

A new interferometric demodulation technique, syntheticheterodyning, has been demonstrated. By synthesizing an FM signal, the system has all the advantages of heterodyne-FM discrimination, i.e., insensitivity to optical power (amplitude) and polarization fluctuations. The need to reset required by phase-lock detection techniques is also eliminated. The FM signal can be produced with a small phase modulator requiring neither a large dynamic range nor high power consumption. Because of the low dynamic range requirement, this technique is compatible with integrated optical modulators. Finally, for multisensor applications one modulator will operate a number of sensors.

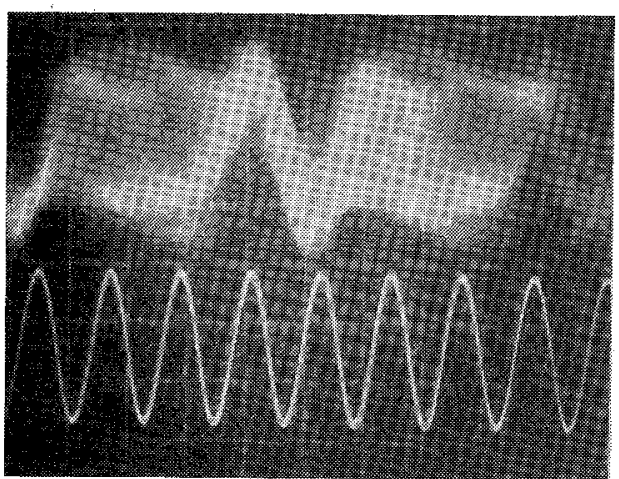

(a)

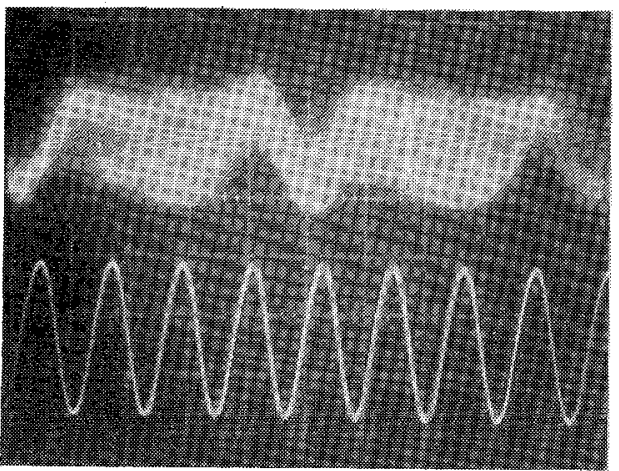

(b)

Fig. 5. Carrier and demodulated output. (a) Without optical attenuation. (b) With approximately 30 percent attenuation.

\section{REFERENCES}

[1] J. H. Cole, T. G. Giallorenzi, and J. A. Bucaro, "Advances in optical fiber sensors," Integ. Opt., p. 269, 1981; also in Proc. Soc. Photo. Opt. Instr. Eng., p. 115.

[2] J. A. Bucaro and J. H. Cole, "Acoustic-optic sensor development," in Proc. EASCON'79, IEEE Publication 79CH1476-1 AES 572, 1979.

[3] D. A. Jackson, A. Dandridge, and S. K. Sheem, "Measurement of small phase shifts using a single mode optical-fiber interferometer," Opt. Lett., vol. 5, p. 139, 1980. 
[4] D. A. Jackson, R. Priest, A. Dandridge, and A. B. Tveten, "Elimination of drift in a single-mode optical fiber interferometer using a piezoelectrically stretched coiled fiber," Appl. Opt., vol. 19. p. $2926,1980$.

[5] I. J. Bush, "Accurate phase measurement system for a fiber optic interferometer," in IEEE OSA Dig. Tech. Pap. Conf. Lasers and Electrooptics, Washington, DC, June 1981.

[6] P. R. Ball and Brian Culshaw, "Digital modulation and phase swept diversity in a coherent multimode fiber system," in Proc. 4th Eur. Conf. Optical Commun., Genova, Italy, Sept. 12-15, 1978, pp. 546-553.

[7] J. H. Cole, T. G. Giallorenzi, and J. A. Bucaro, "Fiber interferometer demodulation and noise," in Proc. FOC'81 EAST Fiber Opt. Commun., Information Gatekeepers, Inc.

[8] P. Shajenko and E. L. Green, "Signal stabilizing of optical interferometric hydrophones by tuning the light source," Appl. Opt., vol. 19, p. $1887,1980$.

[9] A. Olsson, C. L. Tang, and E. L. Green, "Active stabilization of a Michelson interferometer by an electrooptically tuned laser," Appl. Opt., vol. 19, p. 1887, 1980.
James H. Cole, for a photograph and biography, see IEEE J. Quantum Electron., p. 665 .

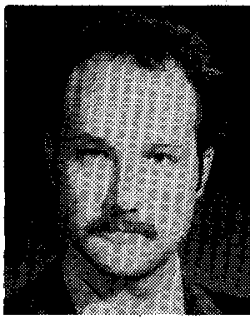

Bruce A. Danver received the B.S. EET degree in electronics from Capitol Institute of Technology in 1981 .

In 1981 he joined Sachs Freeman Associates and is presently doing contract work for the Physical Acoustics Branch at the Naval Research Laboratory. His work consists of electronic design and circuit fabrication required for research investigating fiber sensor transduction mechanisms.

Joseph A. Bucaro, for a photograph and biography, see IEEE J. Quantum Electron., p. 665

\title{
Undersea Fiber Cable Technology
}

\author{
KITSUTARO AMANO, SENIOR MEMBER, IEEE
}

(Invited Paper)

\begin{abstract}
Compared with the conventional coaxial undersea cable systems, an optical fiber undersea cable system has a great technical and economical advantage. It is also suitable for digital transmission. In this paper, the optical fiber undersea cable technology (including optical fiber cables and repeaters), which is now in the research and development stage in several countries, is reviewed.
\end{abstract}

\section{INTRODUCTION}

$\mathrm{C}$ OAXIAL undersea cable systems have been used as one of the major transmission systems in international telecommunication networks over the past 25 years, and its channel capacity has rapidly been increased about ten times per decade with the growth in overseas traffic. However, this system has nearly reached a limit in its ability to increase the capacities of long-haul transoceanic systems from the standpoint of reliability and economy, although additional growth may be permitted with short-haul cable systems. On the other hand, the research and development on optical fiber communication systems progressed remarkably in the 1970's, and a number of systems using $0.85 \mu \mathrm{m}$ wavelength have already been put into commercial services. Recently, the experimental systems using long wavelength and single-mode fibers are now actively being developed worldwide.

Optical fiber undersea cable systems are considered to be a very promising technology to surmount these barriers. At the

Manuscript received September 30, 1981.

The author is with the Research and Development Laboratories, Kokusai Denshin Denwa Company, Ltd., Tokyo, Japan. end of the 1970's the research and development effort was directed toward optical fiber undersea cable systems in the countries which had the experiences of developing the coaxial undersea cable systems. Optical fiber undersea cable systems are recognized to be not only economical, but have improved qualities due to digital transmission capability, and will conform to the future digital communication networks.

Undersea cable systems can be roughly classified into two categories. One is short-haul nonrepeatered systems applicable to interisland, for example. The other is repeatered systems which will be applied to medium-haul transmission routes or international long-haul transmission routes. In this paper, the present status and future trends of long-haul repeatered optical fiber undersea cable technology, which will require the most advanced optical fiber transmission technology, will be reviewed, including a comparison with the conventional coaxial cable systems, the structure of optical fiber cables, and the optical repeater circuits and housing now being developed in Japan and other countries.

\section{An Optical Undersea Cable System}

The principle of transmission systems for undersea cables is basically the same as that for inland long-haul cable systems. However, the fact that the undersea cable is laid into deep sea by a cable ship imposes severe conditions on its mechanical strength. For instance, when the cable is laid under the water some $8000 \mathrm{~m}$ deep, water pressure equivalent to about $800 \mathrm{~atm}$ is applied onto the cable, and also several tons of tension come 